\title{
BMJ Open Study protocol for a randomised trial evaluating the non-inferiority of stepped palliative care versus early integrated palliative care for patients with advanced lung cancer
}

\author{
Kathryn E Post (D) , ${ }^{1}$ Lauren B Heuer, ${ }^{1}$ Arif H Kamal, ${ }^{2}$ Pallavi Kumar, ${ }^{3}$ \\ Madeleine Elyze, ${ }^{1}$ Sarah Griffith, ${ }^{1}$ Jacqueline Han, ${ }^{1}$ Fred Friedman, ${ }^{2}$ \\ Ashley Jackson, ${ }^{3}$ Chardria Trotter, ${ }^{1}$ Rachel Plotke, ${ }^{1}$ Charu Vyas, ${ }^{1}$ Vicki Jackson, ${ }^{1}$ \\ Dustin J Rabideau, ${ }^{1}$ Joseph A Greer, ${ }^{4}$ Jennifer S Temel ${ }^{1}$
}

To cite: Post KE, Heuer LB, Kamal AH, et al. Study protocol for a randomised trial evaluating the non-inferiority of stepped palliative care versus early integrated palliative care for patients with advanced lung cancer. BMJ Open 2022;12:e057591. doi:10.1136/ bmjopen-2021-057591

- Prepublication history for this paper is available online. To view these files, please visit the journal online (http://dx.doi. org/10.1136/bmjopen-2021057591).

Received 21 September 2021 Accepted 19 January 2022

Check for updates

(C) Author(s) (or their employer(s)) 2022. Re-use permitted under CC BY-NC. No commercial re-use. See rights and permissions. Published by BMJ.

${ }^{1}$ Medicine, Massachusetts General Hospital, Boston, Massachusetts, USA

${ }^{2}$ Medicine, Duke University

School of Medicine, Durham,

North Carolina, USA

${ }^{3}$ Medicine, University of

Pennsylvania, Philadelphia,

Pennsylvania, USA

${ }^{4}$ Psychiatry, Massachusetts

General Hospital, Boston,

Massachusetts, USA

Correspondence to

Dr Kathryn E Post;

kepost@partners.org

\section{ABSTRACT}

Introduction Integrating palliative care (PC) early in the illness course for patients with serious cancers improves their outcomes and is recommended by national organisations such as the American Society of Clinical Oncology. However, monthly visits with PC clinicians from the time of diagnosis can be challenging to implement due to the lack of specialty-trained PC clinicians and resources. Therefore, we developed a stepped care model to triage PC service based on patients' needs.

Methods and analysis We are conducting a nonblinded, randomised trial to evaluate the non-inferiority of a stepped PC model compared with an early integrated PC model for improving patients' quality of life (QOL) at 24 weeks (primary outcome). Patients assigned to early integrated PC meet with PC every 4 weeks throughout their illness. Patients assigned to stepped PC have PC visits only at clinically significant points in their illness (eg, cancer progression) unless their QOL decreases, at which time they are 'stepped up' and meet with PC every 4 weeks throughout the remainder of their illness. Secondary aims include assessing whether stepped PC is non-inferior to early integrated PC regarding patientclinician communication about end of life care and length of stay on hospice as well as comparing resource utilisation. Patients are recruited from the Massachusetts General Hospital Cancer Center, Boston, Massachusetts; Duke Cancer Center, Durham, North Carolina and University of Pennsylvania Abramson Cancer Center, Philadelphia, Pennsylvania. The target sample size is 510 patients.

Ethics and dissemination The study is funded by the National Cancer Institute, approved by the Dana-Farber/ Harvard Cancer Center Institutional Review Board and will be reported in accordance with the Consolidated Standards of Reporting Trials statement. We will disseminate results through professional society meetings, peer-reviewed publications and presentations to patient organisations.

Trial registration number NCT03337399.
Strengths and limitations of this study

- This study uses a patient-centred, evidence-based, early integrated palliative care model to improve patient-reported outcomes in those newly diagnosed with advanced lung cancer.

- This study employs a randomised controlled design as well as rigorous intervention fidelity measures to ensure consistent study procedures and intervention delivery across study sites.

- Given the limited availability of palliative care clinicians and clinic resources to implement an early integrated palliative care model in all care settings, this study includes a stepped care arm, which has the potential to be less resource intensive and allow for intervention dissemination.

- The sample is homogenous with respect to patients' cancer type and oncology care at urban academic cancer centres, limiting the ability to generalise the study findings to other populations and care settings.

\section{INTRODUCTION}

Integrating palliative care (PC) and oncology care early in the course of disease for patients with advanced cancer improves their quality of life (QOL), depression symptoms, prognostic understanding and quality of care at the end of life (EOL) as demonstrated by numerous trials over the past decade. ${ }^{1-8}$ These findings were the basis for the 2017 American Society of Clinical Oncology Practice Guidelines, which recommended that patients with advanced cancer in both the inpatient and outpatient settings are offered PC early in the disease trajectory and in conjunction with cancer therapy. ${ }^{9}$ The National Comprehensive Cancer Network also endorses screening 
all oncology patients for their PC needs at their initial oncology consultation as well as at critical time points along their cancer care continuum. ${ }^{10}$

Despite the benefits of integrating palliative and oncology care, adequate numbers of specialty-trained PC clinicians as well as PC infrastructure and resources are lacking to care for all patients diagnosed with advanced cancer. ${ }^{11}{ }^{12}$ Thus, healthcare systems are generally unable to implement this evidence-based, early integrated palliative and oncology care model for their patients in a longitudinal fashion. Studies are needed to examine the potential benefits of alternative approaches to longitudinal PC delivery, such as a stepped PC model in which patients are 'stepped up' to more frequent PC contact based on their clinical needs. Specifically, in a stepped care model, all patients receive care for their condition with a minimum level of required contact with a clinician, and patients are periodically monitored and stepped up to more intensive treatment if the minimal level of engagement with clinicians does not achieve a sufficient health benefit. Stepped care models, which have been successfully used to manage depression, ${ }^{13}$ addiction, ${ }^{14}$ obesity ${ }^{15}$ hypertension, ${ }^{16}$ chronic pain ${ }^{17}$ and distress in patients with cancer, ${ }^{18-20}$ have the potential to achieve similar outcomes and be more cost-effective, feasible and generalisable than traditional models of care. ${ }^{152122}$ Additionally, a stepped PC model is aligned with the shift towards personalised cancer care in that the frequency of PC visits reflects the patients' individual needs throughout their disease course.

The current report outlines the details of an ongoing multisite, randomised controlled trial (RCT) comparing a stepped PC model to an early integrated PC model (entailing monthly contact with a PC clinician) in patients with advanced lung cancer. We seek to demonstrate the non-inferiority of a stepped PC model to the more resource-intensive early integrated PC model, thus establishing a role for this more accessible, adaptable and patient-centred approach to PC.

The primary objective of this study is to determine if stepped $\mathrm{PC}$ is non-inferior to early integrated $\mathrm{PC}$ in improving patients' QOL at 24 weeks as measured by the Functional Assessment of Cancer Therapy-Lung (FACTL). ${ }^{23}$ The secondary aims are to: (1) assess whether stepped PC is non-inferior to early integrated PC with respect to patient-clinician communication about EOL care preferences and length of stay (LOS) on hospice, (2) compare the superiority of stepped PC versus early integrated PC with respect to resource utilisation and (3) determine whether stepped $\mathrm{PC}$ is non-inferior to early integrated PC in improving patients' QOL longitudinally up to 48 weeks.

\section{METHODS AND ANALYSIS \\ Study design}

This is a multisite RCT comparing stepped PC to early integrated $\mathrm{PC}$ in 510 patients with advanced lung cancer

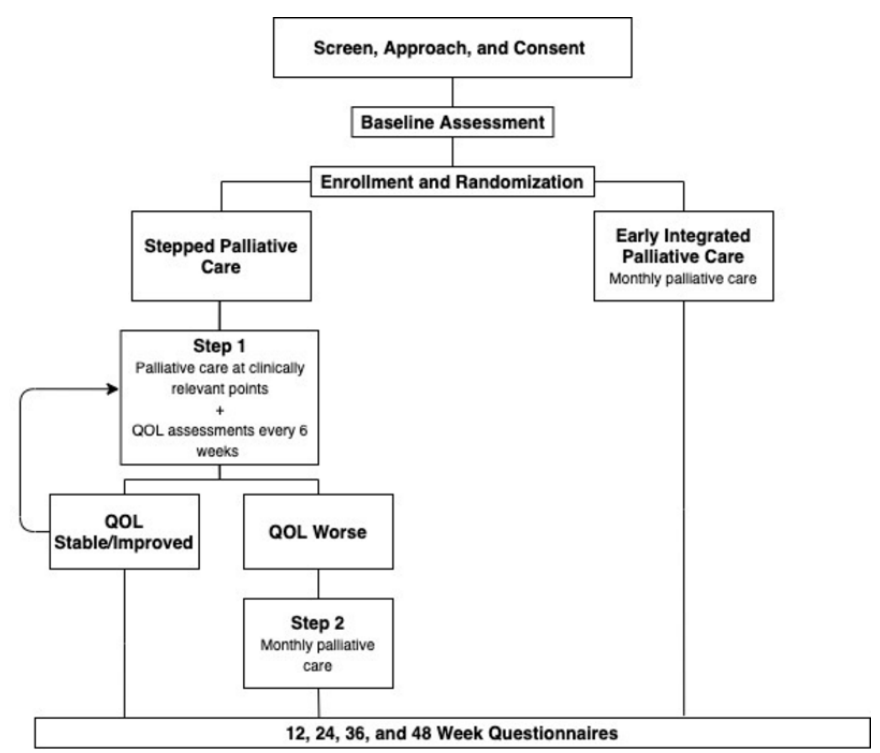

Figure 1 CONSORT flow diagram. CONSORT, Consolidated Standards of Reporting Trials; QOL, quality of life.

at the Massachusetts General Hospital (MGH), Duke Cancer Center and University of Pennsylvania Abramson Cancer Center. The start date of the trial was 1 February 2018 and the estimated completion date is 31 December 2023. The Consolidated Standards of Reporting Trials flow diagram is illustrated in figure 1. The Dana Farber/ Harvard Cancer Center (DF/HCC) Institutional Review Board approved the study prior to initiation.

\section{Patient and public involvement}

We involved patients and the public in the design and conduct of this trial by initially presenting the study design and procedures to the MGH Cancer Outcomes Research \& Education Program (CORE) Patient and Family Advisory Council prior to finalising the study protocol. Additionally, at the conclusion of the trial, we will review the study findings with the CORE Advisory Council as well as disseminate the results through presentations to community organisations, academic institutions and professional societies.

\section{Participant selection}

Eligible patients are $\geq 18$ years old, diagnosed with advanced non-small cell lung cancer (NSCLC), small cell lung cancer (SCLC) or mesothelioma; and treated with non-curative intent and informed of advanced disease within the prior 12 weeks (see figure 2). Patients must also have an Eastern Cooperative Oncology Group (ECOG) Performance Status $\leq 2$, be able to read and respond to questions in English or Spanish and receive their cancer care at a participating site. Patients are excluded if they are already receiving outpatient PC or hospice services since diagnosis of advanced NSCLC, SCLC or mesothelioma. Finally, patients who have cognitive or psychiatric conditions prohibiting study consent or participation, as determined by the treating oncologist, are not eligible for the study. 
Inclusion Criteria:

1. Diagnosed with advanced NSCLC, small cell lung cancer, or mesothelioma, being treated with non-curative intent, and informed of advanced disease within the prior twelve weeks.

2. $\quad$ Eastern Cooperative Oncology Group (ECOG) Performance Status $\leq 2$.

3. Ability to read and respond to questions in English or Spanish

4. Primary cancer care at one of the three participating sites.

5. Age $\geq 18$ years.

Exclusion Criteria:

1. Already receiving outpatient PC or hospice services since diagnosis of advanced NSCLC, small cell lung cancer, or mesothelioma.

2. Cognitive or psychiatric conditions as determined by the treating oncologist to prohibit study consent or participation.

Figure 2 Eligibility criteria. NSCLC, non-small cell lung cancer; PC, palliative care.

\section{Study procedures}

Recruitment

Trained study staff screen the electronic health records (EHR) of all patients presenting to the outpatient thoracic oncology clinic to identify potentially eligible patients for enrolment. Study staff then request permission from the patients' oncology clinicians to approach potentially eligible patients for study participation. Either study staff or an oncology clinician can review with patients the study details, offer study participation and obtain informed consent in person, via telephone or using video conferencing. For patients who speak Spanish, an interpreter or a Spanish-speaking study staff member verbally explains all study procedures and information regarding risks and benefits.

\section{Enrolment and randomisation}

Within 2 weeks of providing informed consent, patients complete baseline demographic and study questionnaires. Once baseline measures are completed, patients are randomised in a 1:1 fashion, stratified by study site (MGH vs Duke vs Penn) and cancer diagnosis (NSCLC vs SCLC and mesothelioma) using a computer-generated randomization schema.

\section{Intervention delivery}

Early integrated PC: patients randomised to early integrated PC are scheduled to meet with a PC clinician within 4 weeks of enrolment and at least every 4 weeks throughout their disease course. $\mathrm{PC}$ visits occur in person or via secure videoconference. If a patient misses a scheduled visit or is unable to be scheduled within 4 weeks of their last PC visit, a PC clinician attempts to call them by telephone to maintain contact at least every 4 weeks and reschedules the visit as soon as possible. The inpatient PC team follows patients who are admitted to a study site hospital.

Stepped PC: patients randomised to stepped PC are scheduled for an initial visit with a PC clinician within 4 weeks of enrolment. During step 1, further visits with a PC clinician are scheduled at clinically significant points in the patient's illness, including within 4 weeks of (1) a change in cancer treatment (due to either progression or toxicity) or (2) hospital discharge. PC visits occur in person or via secure videoconference. After each visit, the PC clinician communicates with the oncology clinician(s) either by telephone, email or in person. If a patient misses a scheduled visit or is unable to be scheduled for a PC visit, the PC clinician attempts to contact them by telephone and reschedules the visit as soon as possible. Patients assigned to stepped PC complete the FACT-L every 6 weeks during the first 18 months of study participation (see table 1 ). Those whose scores decrease by $\geq 10$ points from baseline are 'stepped up' to step 2 and follow the same protocol as those randomised to the early integrated PC arm. Specifically, they meet with a PC clinician at least every 4 weeks for the remainder of their illness and if they are hospitalised.

Table 1 . Study instruments and time points

\begin{tabular}{|c|c|c|c|c|c|c|}
\hline Self-report measure & Baseline & Every 6 weeks* & Week 12 & Week 24 & Week 36 & Week 48 \\
\hline Demographic questionnaire & $x$ & & & & & \\
\hline SCQ & $x$ & & & & & \\
\hline PHQ-9 & $x$ & & $x$ & $x$ & $x$ & $x$ \\
\hline PTPQ & $x$ & & $x$ & $x$ & $x$ & $x$ \\
\hline
\end{tabular}

*Step 1 patients will complete the FACT-L every 6 weeks for up to 18 months from enrolment.

EQ-5D, EuroQol-5 Dimension; FACT-L, Functional Assessment of Cancer Therapy-Lung; PHQ-9, Patient Health Questionnaire-9 ; PTPQ, Prognosis and Treatment Perceptions Questionnaire; SCQ, Self-Administered Comorbidity Questionnaire. 
All study participants in both groups surviving greater than 18 months from enrolment are permitted to decrease the frequency of $\mathrm{PC}$ visits as per their preference and the discretion of their PC and oncology clinicians.

\section{Study questionnaires}

Table 1 lists the self-report questionnaires and the time points at which they are administered.

- At baseline, participants self-report their gender, ethnicity, race, smoking history, with whom they reside as well as the travel time, distance and mode of transportation to the cancer centre. We assess medical comorbidity at baseline with the Self-Administered Comorbidity Questionnaire. ${ }^{24}$

- To measure QOL, patients complete the FACT-L. The FACT-L is a well-validated 36-item tool that assesses five QOL domains, including physical well-being, social/family well-being, emotional well-being and functional well-being as well as a lung cancer-specific subscale $^{23}$ using a 5-point Likert scale ranging from 0 'not at all' to 4 'very much'.

- We assess EOL care preferences via the Prognosis and Treatment Perceptions Questionnaire (PTPQ). The PTPQ is a 9-item tool that assesses patients' illness understanding, communication about prognosis and goals of care as well as discussions and preferences regarding EOL care. $^{25}$

- To assess coping strategies, we administer the Brief Cope, a 28-item questionnaire that assesses methods of coping (eg, active, acceptance, denial) using a 4-point Likert scale ranging from 1 'not at all' to 4 'a lot'. ${ }^{26} 27$ We limit our evaluation to eight coping strategies (16items) of the Brief Cope deemed most relevant for the study (ie, emotional support, positive reframing, active coping, acceptance, self-blame, denial, spiritual coping and behavioural disengagement).

- We evaluate patient depression symptoms via the Patient Health Questionnaire-9 (PHQ-9), a nine-item measure that evaluates symptoms of major depressive disorder according to the criteria of the Diagnostic and Statistical Manual of Mental Disorders. ${ }^{28}$

- We use the EuroQol-5 Dimension (EQ-5D) to measure five dimensions of health including mobility, self-care, usual activities, pain/discomfort and anxiety/depression according to three levels of severity. ${ }^{29} 30$ The EQ-5D also asks patients to rate their health status on a 1-100 visual analogue scale. This tool has been shown to be sensitive to QOL changes in patients with lung cancer. ${ }^{31}$

- At the week 24 primary end point, patients also report their utilisation of any mental health services since diagnosis.

Study staff administer study questionnaires at baseline prior to randomisation and then again at weeks 12, 24, 36 and 48 (with a \pm 2 -week window). As noted above, step 1 patients complete the FACT-L every 6 weeks. Patients may complete questionnaires either in the clinic, on paper at home, via telephone, by secure videoconference or electronically via Research Electronic Data Capture (REDCap),$^{32}$ a Health Insurance Portability and Accountability Act (HIPAA) compliant web-based survey tool.

\section{EHR data}

We are collecting the following information from the EHR: date of birth; cancer type (and genotype if applicable); previous diagnosis of early stage disease; date of diagnosis of advanced lung cancer; smoking pack years; ECOG Performance Status; documentation of advance care directives status; referrals to and LOS on hospice and date and location of death. We are also collecting dates of outpatient and inpatient PC visits; cancer treatment regimens (eg, chemotherapy, immunotherapy, targeted therapy, radiation) and emergency department, hospital, and intensive care unit admissions.

\section{Intervention fidelity}

We are ensuring the fidelity of intervention delivery through consistent training of PC clinicians and reviewing the content of $\mathrm{PC}$ visits.

Training: we standardised the training procedures for site principal investigators (PI) and study staff including the development of a PC intervention guide and study standard operating procedures. The lead study site (MGH) completed a full day, in-person training with the site PIs prior to the study start. The site PIs then trained the participating PC clinicians at their respective institutions using the information they learnt during the in-person training. All participating PC clinicians also reviewed the PC intervention guide and watched training videos developed by the MGH investigative team regarding the intervention delivery and study procedures.

Intervention delivery: PC clinicians complete an electronic survey after each intervention visit to record the topics addressed during the encounter. Two study staff review these electronic surveys quarterly to ensure intervention fidelity and consistency between sites in addressing the domains and topics as specified by the intervention manual. Finally, trained study staff review PC notes in the EHR to ensure adherence to the intervention guide content and provide feedback to clinicians on a quarterly basis. All the site PIs and study staff meet monthly to review intervention delivery and fidelity data.

\section{Outcomes}

Primary outcome

- To determine whether stepped PC is non-inferior to early integrated PC in improving patients' QOL, as measured by the FACT-L at week 24 .

\section{Secondary outcomes}

- To assess the non-inferiority of stepped PC versus early integrated PC with respect to patient-clinician communication about EOL care preferences via the PTPQ at week 48 or the final assessment prior to death.

- To assess whether stepped PC is non-inferior to early integrated PC with respect to hospice LOS. 
- To compare the superiority of stepped PC versus early integrated $\mathrm{PC}$ with respect to resource utilisation.

- To determine whether stepped PC is non-inferior to early integrated PC in improving patients' QOL longitudinally up to 48 weeks as measured by the FACT-L.

\section{Exploratory outcomes}

- To compare the superiority of stepped PC versus early integrated PC with respect to cost-effectiveness.

- To compare coping strategies in patients assigned to stepped PC versus early integrated PC as measured by the Brief Cope at week 24.

- To compare prognostic understanding in patients assigned to stepped $\mathrm{PC}$ versus early integrated PC by analysing relevant items from the PTPQ at week 24.

- To compare depression symptoms in patients assigned to stepped PC versus early integrated PC as measured by the HADS-Depression scale at week 24 .

\section{Safety and adverse events}

Study staff review the PHQ-9 on completion to evaluate for suicidal ideation. If a patient endorses suicidal ideation, the site PI and/or a member of the patient's PC or oncology team are notified and contact the patient to conduct a safety assessment.

Given that this study is a supportive oncology PC intervention trial, we do not anticipate any study-related serious adverse events. We report summaries of study-related non-serious adverse events to the IRB at the continuing reviews. These summaries include types of events, severity and treatment phase. Additionally, the study staff review reasons for study withdrawal by treatment group at weekly meetings.

\section{Data collection and management}

The primary study PI and site PIs oversee all aspects of data collection and management. MGH developed and trained all study staff in the standard operating procedures for data collection, quality control and data extraction. The study staff enter all data abstracted from the EHR as well as all survey data collected from participants in REDCap. Each site maintains a list of patient names and study identification numbers (IDs) saved in a secure file, and participants are identified on study assessments only by study ID to protect confidentiality. Study source documents, including signed informed consent forms, completed eligibility checklists, and participant questionnaires are scanned and stored on secure study site computers.

As this supportive care study has a low risk of studyrelated serious adverse events, we formed a data safety and monitoring committee comprised of MGH investigators to provide additional oversight of data quality and completeness.

\section{Statistical analysis}

We will use intention-to-treat analyses for all randomised subjects. All non-inferiority comparisons will be based on 0.05-level one-sided tests and all superiority comparisons will be based on 0.05-level two-sided tests.

The primary endpoint is to demonstrate the noninferiority of stepped PC versus early integrated PC in improving patients' QOL at 24 weeks, as measured by the FACT-L. The primary endpoint will be analysed between the study groups using a linear regression model controlling for baseline values and demographic and clinical factors and a non-inferiority margin of 4.5 points $(\mathrm{SD}=17.5)$. We will also evaluate the frequency of $\mathrm{PC}$ visits between study arms to determine if the stepped PC model leads to a reduction in PC visits. We will employ linear mixed models of longitudinal data to control for demographic and clinical factors and to account for dependency among means over time when evaluating change in QOL between groups across multiple time points.

The secondary end points of this RCT are to assess whether stepped PC is non-inferior to early integrated PC with respect to communication about EOL care and LOS in hospice. Specifically, we will use the following item from the PTPQ to examine patient reports of discussing EOL care preferences with their clinicians: 'Have you and your doctors discussed any particular wishes you have about the care you would want to receive if you were dying?' We will use either the week 48 assessment or the final assessment before death for this analysis, whichever comes first. We will evaluate differences in patients reporting 'yes' to this item using a Fisher's exact test, and a noninferiority margin of $10 \%$. If there are important imbalances between groups at baseline, we will use a logistic regression model controlling for any demographic and clinical factors that are imbalanced to assess differences between groups.

We will assess the non-inferiority of hospice LOS between stepped PC and early integrated PC. We will use linear regression modelling controlling for selected clinical and demographic factors and a non-inferiority margin of 7 days.

We will compare PC resource utilisation and costeffectiveness between study groups. Costs considered in this analysis will include PC visits, other outpatient care, emergency department use, inpatient care and pharmaceuticals (chemotherapeutics and other pharmaceuticals evaluated separately). ${ }^{33} 34$ To collect outpatient and inpatient hospital costs, we will query the hospital cost accounting system at study sites. ${ }^{35}$ To compare the superiority of stepped PC versus early integrated PC with respect to $\mathrm{PC}$ resource utilisation, we will collect the number and duration of outpatient PC visits from our REDCap database as well as data on the inpatient and telephone PC encounters from the EHR. Both total cost as well as category-specific costs (such as inpatient care, emergency department use and pharmaceuticals) will be evaluated to determine how resource utilisation differs between stepped PC and early integrated PC. Direct healthcare costs and indirect costs (such as time) incurred by patients throughout their life spans while enrolled in this study will be included in this analysis. ${ }^{36}$ We will compare the mean 
number of outpatient and inpatient PC visits between the two groups using a two-sample t test. We will assess the cost-effectiveness of early integrated PC as compared with stepped PC from a societal perspective using the average cost and quality-adjusted life years (QALY) accrued under each study arm. As such, a $\$ 50000$ US dollars QALY will be considered cost effective. ${ }^{37}$

To examine prognostic understanding for noninferiority between stepped PC and early integrated PC at week 24, we will analyse select items from the PTPQ using the appropriate test (eg, Fisher's exact test). Additionally, we will examine coping strategies (Brief Cope) and depression symptoms (PHQ-9) at week 24 between groups using linear regression models controlling for baseline values and selected demographic and clinical factors. Linear mixed models will also be used as described above to examine changes in these outcomes between groups across multiple time points.

We will explore potential moderators of the interventions to ensure generalisability and identify whether certain groups benefit more from one of the two PC models. We will create interaction terms for the regression and linear mixed models to examine whether differences in outcomes are moderated by patient factors (age, gender and race) or study site.

Finally, we will employ multiple imputation methods when data can reasonably be assumed to be missing at random. In settings where data are likely missing not at random (eg, due to progressive illness), we will employ pattern mixture modelling or terminal decline joint modelling to address missing data.

\section{Sample size}

For the primary outcome in our previous trials of early integrated $\mathrm{PC}$, we assessed the change in QOL, as measured by the FACT-L from baseline to week $12 .{ }^{638}$ However, for this trial, we chose to focus on week 24 as life expectancy for patients with advanced lung cancer has improved in recent years. ${ }^{390}$ With 188 patients per group, we will have $80 \%$ power to demonstrate the non-inferiority of stepped PC versus early integrated $\mathrm{PC}$ in improving patientreported QOL as measured by the FACT-L and a noninferiority margin of 4.5 points $(\mathrm{SD}=17.5)$. To account for potential missing data and ensure adequate power to assess for non-inferiority, we increased our sample size to 255 per group for a total sample size of 510 participants.

\section{Limitations}

The current trial has several potential limitations. First, we are only enrolling patients with advanced lung cancer receiving care at three large academic medical centres, limiting generalisation of the results to patients with different cancer types or stages of disease as well as those receiving treatment in other oncology care settings. However, existing data support early integrated PC in patients with advanced lung cancer, ${ }^{6} 1938$ making this an ideal population in which to compare different PC models. Second, we are only enrolling English and
Spanish-speaking patients due to the availability of study questionnaires in these languages. In future studies, investigators could consider study procedures to enrol patients who speak languages other than English or Spanish. Third, both the participating study clinicians and patients are aware of the study group assignments, potentially introducing bias. However, the frequency and timing of intervention visits precluded blinding PC clinicians or patients to the study group assignments. Finally, we do not prevent patients in the stepped PC group from having additional appointments with their PC clinicians if requested by either the patient or the clinician, which could also influence study findings. However, denying PC services for patients with advanced cancer would neither be feasible nor acceptable.

\section{ETHICS AND DISSEMINATION}

This trial was approved by the DF/HCC IRB and is being conducted in compliance with the approved protocol. We are obtaining informed consent either in person, verbally via telephone or via secure videoconferencing technology. Patients who provide informed consent verbally receive a copy of the written consent form. All documents relating to study participants are confidential. Participant data are deidentified and stored in a HIPAA-compliant manner. All significant modifications to the study protocol have and will be submitted to the DF/HCC IRB for approval and communicated to study staff at all sites as well as to patients as indicated.

We will present the study findings through multiple outlets including national conferences, peer-reviewed publications, social media and community organisations. A study description and summary of the results will also be available on ClinicalTrials.gov. Only the study staff have access to the study database, however, access can be considered via a data usage agreement with the DF/HCC IRB. There are no plans for professional writers for the final manuscript. If a study patient expresses an interest in the study findings, the study staff will provide an abstract of study findings once data collection is complete.

\section{Current trial status}

We began recruitment of participants on 12 February 2018. As of 10 September 2021, 384 patients have enrolled. We placed the study on a temporary recruitment pause in March of 2020 due to the COVID-19 pandemic and resumed recruitment in July 2020.

Acknowledgements We thank the study patients for their time and dedication to this research study.

Contributors JST, JAG and VJ contributed to the study conception and design. The protocol was developed and written by JST, JAG and VJ. KEP, LBH, ME, SG, JH, FF, AJ provided substantial contributions to the acquisition of data for the study. The first draft of the manuscript was written by KEP and LBH. JST, JAG, AHK, PK, ME, $\mathrm{SG}, \mathrm{JH}, \mathrm{CT}, \mathrm{RP}, \mathrm{CV}$ and DJR contributed critically important revisions on previous versions of the manuscript. All authors read and approved the final manuscript.

Funding This study is supported by a R01 grant from the National Cancer Institute of the National Institutes of Health (R01CA215188; Temel). 
Competing interests None declared.

Patient and public involvement Patients and/or the public were involved in the design, or conduct, or reporting, or dissemination plans of this research. Refer to the Methods section for further details.

Patient consent for publication Not applicable.

Provenance and peer review Not commissioned; peer reviewed for ethical and funding approval prior to submission.

Open access This is an open access article distributed in accordance with the Creative Commons Attribution Non Commercial (CC BY-NC 4.0) license, which permits others to distribute, remix, adapt, build upon this work non-commercially, and license their derivative works on different terms, provided the original work is properly cited, appropriate credit is given, any changes made indicated, and the use is non-commercial. See: http://creativecommons.org/licenses/by-nc/4.0/.

\section{ORCID iD}

Kathryn E Post http://orcid.org/0000-0002-1777-5158

\section{REFERENCES}

1 Rabow MW, Dibble SL, Pantilat SZ, et al. The comprehensive care team: a controlled trial of outpatient palliative medicine consultation. Arch Intern Med 2004;164:83-91.

2 Bakitas MA, Tosteson TD, Li Z, et al. Early versus delayed initiation of concurrent palliative oncology care: patient outcomes in the enable III randomized controlled trial. J Clin Oncol 2015;33:1438-45.

3 Bakitas M, Lyons KD, Hegel MT, et al. Effects of a palliative care intervention on clinical outcomes in patients with advanced cancer: the project enable II randomized controlled trial. JAMA 2009;302:741-9.

4 Dionne-Odom JN, Azuero A, Lyons KD, et al. Benefits of early versus delayed palliative care to informal family caregivers of patients with advanced cancer: outcomes from the enable III randomized controlled trial. J Clin Oncol 2015;33:1446-52.

5 Zimmermann C, Swami N, Krzyzanowska M, et al. Early palliative care for patients with advanced cancer: a cluster-randomised controlled trial. Lancet 2014;383:1721-30.

6 Temel JS, Greer JA, Muzikansky A, et al. Early palliative care for patients with metastatic non-small-cell lung cancer. $N$ Engl J Med 2010;363:733-42.

7 Ferrell B, Sun V, Hurria A, et al. Interdisciplinary palliative care for patients with lung cancer. J Pain Symptom Manage 2015;50:758-67.

8 Greer JA, Jackson VA, Meier DE, et al. Early integration of palliative care services with standard oncology care for patients with advanced cancer. CA Cancer J Clin 2013;63:349-63.

9 Ferrell BR, Temel JS, Temin S, et al. Integration of palliative care into standard oncology care: American Society of clinical oncology clinical practice guideline update. J Clin Oncol 2017;35:96-112.

10 Dans M, Kutner JS, Agarwal R. NCCN clinical practice guidelines in oncology, palliative care (version 2.2021). National comprehensive cancer network. Available: https://www.nccn.org/professionals/ physician gls/pdf/palliative.pdf [Accessed 5 Apr 2021].

11 Hui D, Elsayem A, De la Cruz M, et al. Availability and integration of palliative care at US cancer centers. JAMA 2010;303:1054-61.

12 Lupu D, American Academy of Hospice and Palliative Medicine Workforce Task Force. Estimate of current hospice and palliative medicine physician workforce shortage. J Pain Symptom Manage 2010;40:899-911.

13 van Straten A, Hill J, Richards DA, et al. Stepped care treatment delivery for depression: a systematic review and meta-analysis. Psychol Med 2015;45:231-46.

14 Breslin FC, Sobell MB, Sobell LC, et al. Toward a stepped care approach to treating problem drinkers: the predictive utility of within-treatment variables and therapist prognostic ratings. Addiction 1997;92:1479-89.

15 Jakicic JM, Tate DF, Lang W, et al. Effect of a stepped-care intervention approach on weight loss in adults: a randomized clinical trial. JAMA 2012;307:2617-26.

16 Azizi M, Sapoval M, Gosse P, et al. Optimum and stepped care standardised antihypertensive treatment with or without renal denervation for resistant hypertension (DENERHTN): a multicentre, open-label, randomised controlled trial. Lancet 2015;385:1957-65.

17 Kroenke K, Krebs EE, Wu J, et al. Telecare collaborative management of chronic pain in primary care: a randomized clinical trial. JAMA 2014;312:240-8.

18 Krebber AMH, Jansen F, Witte BI, et al. Stepped care targeting psychological distress in head and neck cancer and lung cancer patients: a randomized, controlled trial. Ann Oncol 2016;27:1754-60.

19 Jansen F, Krebber AMH, Coupé VMH, et al. Cost-Utility of stepped care targeting psychological distress in patients with head and neck or lung cancer. J Clin Oncol 2017;35:314-24.

20 Singer S, Danker H, Briest S, et al. Effect of a structured psychooncological screening and treatment model on mental health in cancer patients (stepped care): study protocol for a cluster randomized controlled trial. Trials 2014;15:482.

21 Salloum A, Wang W, Robst J, et al. Stepped care versus standard trauma-focused cognitive behavioral therapy for young children. $J$ Child Psychol Psychiatry 2016;57:614-22.

22 Fokkenrood HJP, Scheltinga MRM, Koelemay MJW, et al. Significant savings with a stepped care model for treatment of patients with intermittent claudication. Eur J Vasc Endovasc Surg 2014;48:423-9.

23 Cella D. The functional assessment of cancer Therapy-Lung and lung cancer Subscale assess quality of life and meaningful symptom improvement in lung cancer. Semin Oncol 2004;31:11-15.

24 Sangha O, Stucki G, Liang MH, et al. The self-administered comorbidity questionnaire: a new method to assess comorbidity for clinical and health services research. Arthritis Rheum 2003;49:156-63.

25 El-Jawahri A, Traeger L, Park ER, et al. Associations among prognostic understanding, quality of life, and mood in patients with advanced cancer. Cancer 2014;120:278-85.

26 Carver CS. You want to measure coping but your protocol's too long: consider the brief cope. Int J Behav Med 1997;4:92-100.

27 Hagan TL, Fishbein JN, Nipp RD, et al. Coping in patients with incurable lung and gastrointestinal cancers: a validation study of the brief cope. J Pain Symptom Manage 2017;53:131-8

28 Kroenke K, Spitzer RL, Williams JB. The PHQ-9: validity of a brief depression severity measure. J Gen Intern Med 2001;16:606-13.

29 Chouaid C, Agulnik J, Goker E, et al. Health-Related quality of life and utility in patients with advanced non-small-cell lung cancer: a prospective cross-sectional patient survey in a real-world setting. $J$ Thorac Oncol 2013;8:997-1003.

30 EuroQol Group. EuroQol--a new facility for the measurement of health-related quality of life. Health Policy 1990;16:199-208.

31 O'Brien MER, Ciuleanu T-E, Tsekov H, et al. Phase III trial comparing supportive care alone with supportive care with oral topotecan in patients with relapsed small-cell lung cancer. J Clin Oncol 2006;24:5441-7.

32 Harris PA, Taylor R, Thielke R, et al. Research electronic data capture (REDCap)--a metadata-driven methodology and workflow process for providing translational research informatics support. J Biomed Inform 2009;42:377-81.

33 Greer JA, Tramontano AC, McMahon PM, et al. Cost analysis of a randomized trial of early palliative care in patients with metastatic nonsmall-cell lung cancer. J Palliat Med 2016;19:842-8.

34 Hay JW, Smeeding J, Carroll NV, et al. Good research practices for measuring drug costs in cost effectiveness analyses: issues and recommendations: the ISPOR Drug Cost Task Force report--Part I. Value Health 2010;13:3-7.

35 Pronovost P, Angus DC. Cost reduction and quality improvement: it takes two to tango. Crit Care Med 2000;28:581-3.

36 Drummond MF, Sculpher MJ, Claxton K. Methods for economic evaluation of health care programmes. 4th ed. New York, NY: Oxford University Press, 2015.

37 Neumann PJ, Cohen JT, Weinstein MC. Updating cost-effectiveness-the curious resilience of the $\$ 50,000$-per-QALY threshold. $N$ Engl J Med 2014;371:796-7.

38 Temel JS, Greer JA, El-Jawahri A, et al. Effects of early integrated palliative care in patients with lung and $\mathrm{Gi}$ cancer: a randomized clinical trial. J Clin Oncol 2017;35:834-41.

39 Hirsch FR, Suda K, Wiens J, et al. New and emerging targeted treatments in advanced non-small-cell lung cancer. Lancet 2016;388:1012-24.

40 Howlader N, Forjaz G, Mooradian MJ, et al. The effect of advances in lung-cancer treatment on population mortality. N Engl J Med 2020;383:640-9. 